\title{
La importancia de la ética, el respeto a la autodeterminación y los derechos fundamentales de los pueblos indígenas en aislamiento en la antropología
}

\author{
The Importance of Ethics, Respect for Self-determination \\ and the Fundamental Rights of Isolated Indigenous People in \\ Anthropology
}

\author{
Beatriz Huertas Castillo \\ https://orcid.org/0000-0002-5104-9256 \\ Antropóloga y Consultora Independiente \\ beahuertasc@gmail.com
}

Recibido: 04/11/2019 - Aceptado: 13/04/2020 - Publicado: 12/03/2021

\section{Resumen}

Este artículo aborda el resurgimiento de planteamientos, desde la antropología, a favor de que los Estados tomen la iniciativa de establecer relaciones directas y permanentes con pueblos indígenas, o parte de ellos, que viven en aislamiento. Analiza el contexto y los argumentos utilizados por los impulsores de esta posición en la actualidad, así como los impactos que iniciativas similares han tenido en pueblos indígenas. En contraposición, expone el proceso histórico, los principios y los avances del enfoque de respeto a la autodeterminación y los territorios de estos pueblos. En esa línea, apuesta por una antropología respetuosa de las decisiones de los pueblos en aislamiento sobre su forma de vida y el nivel de interacción que desean tener con el entorno. A la vez, plantea la necesidad de contribuir a garantizar la seguridad jurídica y la protección de sus territorios, implementar medidas preventivas en salud y anteponer el respeto de sus derechos fundamentales a intereses personales, gubernamentales, económicos u otros al momento de tomar decisiones que los afecten.

\section{Palabras claves}

Pueblos indígenas en aislamiento; autodeterminación; seguridad jurídica y protección territorial; contacto controlado; contacto forzado. 


\section{Abstract}

This article addresses the resurgence of anthropological approaches promoting that states taking the initiative to establish direct and permanent relationships with indigenous peoples, or part of them, who live in isolation. It analyzes the context and the arguments used by the promoters of this position today, as well as the impacts that similar initiatives have had on indigenous peoples. In contrast, it exposes the historical process, the principles and the advances of the approach of respect for selfdetermination and for the territories of these peoples. Along these lines, it is committed to an anthropology that is respectful of the decisions of peoples in isolation about their way of life and the level of interaction they wish to have with the broader environment. At the same time, it raises the need to contribute to guarantee legal security and the protection of their territories, to implement preventive health measures and to put respect for their fundamental rights before personal, governmental, economic or other interests when making decisions that affect them.

\section{Keywords}

Isolated indigenous people; self-determination; legal security and territorial protection; controlled contact; forced contact.

Citar como: Huertas Castillo B. (2021). La Importancia de la Ética, el Respeto a la Autodeterminación y los Derechos Fundamentales de los Pueblos Indígenas en Aislamiento en la Antropología. Revista de antropología núm. 8: 86 - 104. http://dx.doi.org/10.15381/antropologia.v0i8.19809

\section{INTRODUCCIÓN}

Desde el año 2014, en el contexto de la mayor exposición de integrantes de algunos pueblos indígenas en aislamiento ${ }^{1}$ a interacciones con poblaciones vecinas en el Perú y Brasil, algunos investigadores, principalmente del campo de la antropología, han venido promoviendo posturas y acciones orientadas a que los Estados aceleren el establecimiento de relaciones directas y sostenidas, no solo con estas personas, sino con los pueblos en aislamiento en general. En algunos casos, este planteamiento, también conocido como "contacto forzado" o "contacto controlado", vendría acompañado del interés de realizar estudios antropológicos y lingüísticos de estos pueblos en base a metodologías directas de recopilación de información. De esta manera, su decisión de distanciarse de la sociedad, su forma de vida, los riesgos que el llamado contacto forzado ha tenido históricamente sobre su integridad física, sociocultural y territorial, y sus derechos fundamentales no son considerados ni respetados. A fin de justificar esta posición, sus promotores han tergiversado el enfoque de respeto a la autodeterminación y garantía de los derechos de los pueblos en aislamiento,

${ }^{1}$ Pueblos mashco piro del río Alto Madre de Dios (Perú) y korubo de la Tierra Indígena Valle del Yavarí y pueblo del Xinane, de la cuenca del Yurúa (Brasil). Este último terminó estableciendo relaciones directas con funcionarios de la Fundación Nacional del Indio, de Brasil, y se trasladó a las proximidades de la Base de vigilancia de esta institución gubernamental en el río Xinane. 
el cual viene siendo impulsado desde los años ochenta por organizaciones indígenas, estudiosos, activistas y organismos internacionales de defensa de los derechos humanos, como la Organización de las Naciones Unidas y la Comisión Interamericana de Derechos Humanos. Al respecto, la autodeterminación o derecho que estos pueblos tienen de decidir sobre su forma de vida y nivel de interacción con otras personas no solo ha sido simplificada únicamente a lo que se ha denominado la postura del "leave them alone" ("déjenlos en paz"\} (Walker y Hill, 2015, p. 1061), sino que ha sido desvirtuada y caricaturizada al atribuírsele percepciones en las que el aislamiento es realzado, fomentado, "fetichizado" o admirado exageradamente (Shepard, 2016, p. 135).

Otro fundamento de la posición a favor del contacto forzado cae en la sobrevaloración de la capacidad del Estado de manejar satisfactoriamente procesos impuestos de interacción directa y sostenida, y sus efectos sobre la salud, el acceso al territorio y la subsistencia, entre otros aspectos de la vida de los pueblos involucrados (Walker y Hill, 2015). Históricamente, la integración forzada ha tenido efectos devastadores para los pueblos indígenas en aislamiento debido al desencadenamiento de conflictos, expansión de enfermedades, muertes masivas, desestructuración demográfica, social, económica y política, y pérdida territorial. El nuevo impulso que, incluso desde el propio Estado, cobró en el país esta antigua práctica en los últimos años, flexibilizó los principios y estándares de protección de estos pueblos, que habían sido conquistados para ellos con mucho esfuerzo por organizaciones indígenas y activistas, a lo largo de más de tres décadas. Dicha flexibilización favorece la disposición de sus territorios con fines de explotación de los recursos naturales y la promoción de proyectos de inversión en ellos, los cuales forman parte de las prioridades gubernamentales.

Este artículo desarrolla las dos posturas contrapuestas y toma posición por el respeto a la autodeterminación de los pueblos en aislamiento y a sus derechos fundamentales, de la mano de la adopción de medidas de prevención frente al riesgo epidemiológico y de conflictos con poblaciones vecinas. El texto se divide en cinco partes: en la primera se explica a quienes nos referimos cuando hablamos de pueblos en aislamiento, así como las limitaciones de este término para comprender sus características socioculturales a cabalidad desde la mirada antropológica. Seguidamente, se desarrolla la historia de la defensa y protección de estos pueblos en el Perú, distinguiendo tres etapas bajo el criterio de las dimensiones y la complejidad que fueron adquiriendo las iniciativas, acciones y demandas desarrolladas a lo largo de las tres décadas transcurridas desde que este proceso se inició. La autodeterminación y el territorio, principios que orientaron la defensa de los pueblos en aislamiento, son abordados en la tercera parte, para luego seguir con los fundamentos e implicancias del contacto forzado, controlado y de propuestas similares sobre estos pueblos. Algunas pautas generales para abordar los retos que encierran situaciones de intensificación de interacciones 
por pueblos en aislamiento son mencionadas en el quinto acápite, para luego dar paso a las consideraciones finales.

\section{Aspectos conceptuales}

En el Perú, el término pueblos en aislamiento se viene utilizando desde los años noventa para hacer referencia a aquellos pueblos, o parte de ellos, que rechazan o limitan sus interacciones con personas ajenas a sus propias colectividades. Estudios etnohistóricos realizados sobre los pueblos indígenas de la Amazonía sur del Perú (Zarzar, 1983; Álvarez Lobo, 1998; Huertas, 2002) demuestran que los antecesores de varias de estas poblaciones interactuaban con miembros de su conjunto mayor u otros pueblos, manteniendo relaciones de parentesco, de intercambio de bienes, de conflicto, de alianza u otros. Posteriormente, con la irrupción de la economía del caucho, ocurrida a fines del siglo XIX, se desencadenaron persecuciones, matanzas, relaciones de esclavitud, abusos de diferente índole y expansión de enfermedades que diezmaron a la población. Estas condiciones de agresión extrema, sumadas a factores internos, como el resquebrajamiento de las relaciones intra e interétnicas, llevaron a numerosas poblaciones indígenas a apartarse de las fronteras de la violencia para sobrevivir.

Estas poblaciones se desplazaron hacia lugares distantes, en cabeceras de ríos y quebradas, donde adoptaron una forma de vida y sistemas de producción basados en una alta movilidad, condicionados por la estacionalidad climática y las épocas de reproducción de los recursos del bosque. En los años posteriores, la invasión de los espacios que habitaban continuó a través del tráfico de pieles, de la explotación de hidrocarburos y madera, y en los últimos años, del narcotráfico. De esta manera, en el caso de los pueblos de la Amazonía sur del Perú, el aislamiento puede ser entendido como una estrategia de sobrevivencia asumida en momentos históricos de intensa agresión externa que con el paso del tiempo adquirió nuevas formas e involucró a nuevos actores (Huertas, 2012). También existen poblaciones que han adoptado el aislamiento en las últimas décadas (fines del siglo XX), como los mbya guaraní, de Paraguay, quienes luego de vivir articulados a la sociedad envolvente, decidieron rechazar la presencia y servicios del Estado, así como de foráneos en sus comunidades, limitando sus relaciones únicamente a algunos líderes de su conjunto etnolingüístico mayor. En estos casos, el aisla miento es relativamente reciente y demuestra, nuevamente, que las relaciones de interacción sostenida pueden revertir a una interacción muy limitada.

Existen diferentes niveles de aislamiento o limitación de interacciones, desde poblaciones que practican una serie de estrategias para ocultar sus rastros, o que rechazan con hostilidad la presencia externa en sus territorios, hasta las que se aproximan a poblados vecinos para obtener objetos manufacturados que les son útiles, exponiéndose en alguna medida a ser vistos. En todos los casos, 
rehúyen las interacciones sostenidas con otras personas, ya sea integrantes de sus conjuntos etnolingüísticos mayores o foráneos. Esta actitud respondería a varios factores, como su temor a ser agredidos o a adquirir enfermedades, así como su percepción del otro y de su forma de vida en contraposición con la propia.

El término pueblos no contactados resulta inadecuado puesto que da a entender que existen sociedades que han vivido completamente al margen de lo que ocurre en su medio y estancadas en el tiempo, lo cual no ha existido ni existe en la realidad. Los términos pueblos en aislamiento o pueblos en contacto inicial tampoco denotan la complejidad de las características socioculturales y sistemas organizativos, económicos y simbólicos de estos pueblos; su uso en el presente artículo se enmarca solo en dos criterios fundamentales: enfatizar su decisión de limitar sus interacciones con la población del entorno y la situación de vulnerabilidad, principalmente inmunológica, que presentan y pone su sobrevivencia en riesgo. De la misma manera, como se ha visto, utilizamos el término interacción en vez de contacto, dado el papel activo que desempeña la población en aislamiento en el tipo de relaciones que mantiene con el entorno, así estas sean muy limitadas (Shinai Serjali, 2004).

La Amazonía peruana está habitada por una diversidad de pueblos o segmentos de pueblos en aislamiento. Se localizan en zonas de cordillera y en las fronteras con Brasil y Ecuador, pudiendo habitar también la frontera con Bolivia. La mayor parte está clasificada en las familias lingüísticas pano y arawak, habiendo otros pueblos cuya pertenencia lingüística no ha podido ser definida. El Ministerio de Cultura ha reconocido oficialmente 20 pueblos en aislamiento y seis en contacto inicial.

\section{BREVE historia DE LA DEFENSA Y LA PROTECCIÓN DE LOS PUEBLOS EN aislamiento en el Perú}

Desde mediados de los ochenta, los propios pueblos y organizaciones indígenas, con el apoyo de organizaciones nacionales e internacionales aliadas, han promovido la defensa y protección de los pueblos en aislamiento con el objetivo de garantizar su sobrevivencia. Los lineamientos que orientaron este quehacer fueron la seguridad jurídica y protección territorial, así como el respeto al modo de vida y decisiones de estos pueblos. En base al análisis de las dimensiones que fue alcanzando este proceso, tanto en términos temáticos, geográficos y de involucramiento de actores, se puede distinguir tres etapas: el inicio, la expansión y una tercera etapa en la que surgen, a la vez, y paradójicamente, propuestas de protección vanguardistas y propuestas que habían sido descartadas años atrás por sus nefastas consecuencias sobre los pueblos en aislamiento, como el llamado "contacto controlado" o forzado. Las dos primeras etapas fueron conducidas por las organizaciones indígenas, mientras que el Estado recién entró a tallar de manera sostenida en la tercera. 
La importancia de la ética, el respeto a la autodeterminación y los derechos fundamentales de los pueblos indígenas en aislamiento en la antropología

\section{A. LA ETAPA INICIAL (1984-2002)}

La tragedia que el pueblo nahua enfrentó a mediados de los años ochenta, como consecuencia del contacto forzado, marcó el hito con el que se inició la historia de la defensa y protección de los pueblos en aislamiento en el Perú. En 1984, en un contexto de búsqueda de contactos forzados por el Instituto Lingüístico de Verano (ILV), de actividad maderera y de operaciones hidrocarburíferas de la empresa Shell en las cabeceras del río Mishagua, se produjo la captura de cuatro miembros del pueblo nahua, que habitaba la zona. A ello siguieron traslados de sus integrantes hacia el poblado próximo de Sepahua. Poco después, aproximadamente el 50\% de sus miembros falleció debido a la expansión de enfermedades respiratorias, que les fueron transmitidas por la población con la que entraron en contacto directo, frente a las cuales no habían desarrollado defensas inmunológicas (Helberg y Reynoso, 1986). Enseguida se produjo la llegada masiva de madereros a su territorio, mientras que la empresa Shell continuó con sus operaciones. Mientras el gobierno trataba de silenciar esta nueva tragedia de un pueblo indígena que había estado viviendo en aislamiento, organizaciones de la sociedad civil denunciaron los hechos a nivel internacional y exigieron atención médica para sus miembros, así como la protección de sus territorios.

El Consejo Machiguenga del Río Urubamba (COMARU) y la Federación Nativa del Río Madre de Dios y Afluentes (FENAMAD) gestionaron ante el Estado el reconocimiento del territorio del pueblo nahua y de otros pueblos en aislamiento y contacto inicial que colindan con este, logrando en 1990 la creación de la Reserva Territorial Kugapakori-Nahua (Chirif y García, 2007). Esta fue la primera reserva establecida a favor de pueblos en aislamiento y contacto inicial en el Perú, y se basó en la Ley de Comunidades Nativas y Desarrollo Agrario de la Selva y de Ceja de Selva (22175).

A mediados de los noventa, la Asociación Interétnica de Desarrollo de la Selva Peruana (AIDESEP), organización indígena amazónica de nivel nacional, propuso al Estado la creación de cuatro reservas territoriales para pueblos en aislamiento en el departamento de Ucayali: Murunahua, Mashco Piro, Isconahua y Kakataibo, y elaboró los estudios técnicos respectivos ${ }^{2}$. Las tres primeras fueron creadas entre 1997 y 1998 a través de normas regionales, mientras que la cuarta aún no ha sido establecida.

En 1996, ante el otorgamiento de derechos de explotación de hidrocarburos por el gobierno a favor de la empresa Mobil Exploration and Producing sobre territorios de pueblos en aislamiento en el norte de Madre de Dios, la Federación Nativa del Río Madre de Dios y Afluentes (FENAMAD) llevó a cabo acciones de incidencia ante la empresa petrolera y el Estado para proteger a estos pueblos frente a los peligros que suponían las operaciones petroleras. Seguidamente,

${ }^{2}$ Para este fin, contó con el apoyo financiero de la cooperación danesa a través del Grupo Internacional de Trabajo sobre Asuntos Indígenas (IWGIA). 
realizó los estudios y gestiones para crear una reserva a favor de estos pueblos ${ }^{3}$, lo cual se concretó en el 2002 con el establecimiento oficial de la Reserva Territorial Madre de Dios, tras una intensa confrontación con empresas madereras.

\section{B. ETAPA DE EXPANSIÓN DE LAS ACCIONES DE DEFENSA Y PROTECCIÓN (2003- 2010)}

En diciembre de 2002, durante su XIX congreso nacional, AIDESEP y sus federaciones base crearon el Programa Nacional de Defensa y Protección de Pueblos Indígenas en Aislamiento y Contacto Inicial. Con el apoyo técnico de este programa, entre los ańos 2003 y 2005, la organización solicitó al Estado la creación de cuatro nuevas reservas para pueblos en aislamiento, esta vez en la región Loreto (Napo-Tigre, Yavarí-Tapiche, Yavarí Mirim y Kapanawa), y se realizaron los estudios técnicos correspondientes. Por su parte, la organización no gubernamental Instituto del Bien Común, junto a la Federación Nativa de Comunidades Kakataibo (FENACOCA), realizó estudios complementarios de la propuesta de reserva territorial Kakataibo y volvió a solicitar al Estado su establecimiento.

Paralelamente, AIDESEP elaboró una propuesta de régimen especial para la protección de pueblos en aislamiento y contacto inicial. Tal como señalan Chirif y García (2007), esta iniciativa proponía darle institucionalidad al tratamiento de estos pueblos, prohibir en las reservas territoriales el asentamiento de poblaciones distintas a las que habitan en ellas y vetar cualquier intento de entregar parte o la totalidad del área bajo contratos de extracción de recursos, entre otros. En esa misma línea, en noviembre del 2005, a través de una resolución ministerial, se aprobó el Informe Defensorial 101 sobre pueblos en aislamiento y contacto inicial. Este documento planteó recomendaciones a cada sector del Estado involucrado en la protección de estos pueblos, entre ellas, aprobar una iniciativa legislativa que establezca una categoría especial para la intangibilidad de las reservas territoriales. Al año siguiente se promulgó la "Ley para la protección de pueblos indígenas u originarios en situación de aislamiento y en situación de contacto inicial" (Ley $\mathrm{N}^{\circ}$ 28736). Si bien tal como fue recomendado por la Defensoría del Pueblo, esta ley estableció una nueva categoría, la de reserva indígena, y dispuso la creación de un régimen especial transectorial de protección, debilitó drásticamente los derechos territoriales de los pueblos en aislamiento y contacto inicial, y abrió las puertas de las reservas indígenas para la explotación de recursos naturales mediante el argumento de la necesidad pública.

Este periodo también se caracterizó por el despliegue de esfuerzos desde la sociedad civil para defender la integridad de la Reserva Territorial Kugapakori Nahua frente a la superposición del lote 88 o Proyecto Gas de Camisea a parte importante de su superficie. En el 2005, el incremento de conflictos entre

${ }^{3}$ En este caso, la organización indígena también contó con el apoyo de la cooperación danesa. 
madereros e indígenas en aislamiento en el río Las Piedras, en Madre de Dios, y la negativa de las autoridades locales a admitir las acciones legales presentadas frente a ello, llevaron a FENAMAD a pedir apoyo técnico a AIDESEP para tomar acciones legales en el ámbito internacional. Fue así como ese mismo año se presentó una solicitud de medidas cautelares ante la Comisión Interamericana de Derechos Humanos (CIDH) para que el Estado protegiera a los pueblos en aislamiento frente a la invasión de sus territorios por madereros ${ }^{4}$.

En el 2006 AIDESEP y las organizaciones regionales FENAMAD y la Organización Regional AIDESEP-Ucayali (ORAU) iniciaron la construcción y funcionamiento de puestos de control y vigilancia en las zonas de acceso a las reservas territoriales Madre de Dios, Isconahua, Murunahua y Mashco Piro $^{5}$. Ese mismo año, la realización de un foro internacional sobre pueblos en aislamiento y contacto inicial en Santa Cruz de la Sierra ${ }^{6}$, Bolivia, facilitó el encuentro de los líderes y lideresas indígenas de los siete países de América del Sur con presencia de estos pueblos. El diálogo entre ellos conllevó la decisión de crear una plataforma de coordinación entre organizaciones indígenas que adoptó el nombre de Comité Indígena Internacional para la Protección de los Pueblos en Aislamiento y Contacto Inicial de la Amazonía, el Gran Chaco y la Región Oriental de Paraguay (CIPIACI). Sus acciones se centraron en la incidencia ante la Organización de las Naciones Unidas (ONU), la CIDH y la elaboración de las Directrices de la ONU para la protección de los pueblos mencionados, proceso que fue conducido por el Alto Comisionado de la ONU para los Derechos Humanos.

\section{Corredores terRitoriales, EL PAPEL DEL ESTADO Y LA POSTURA DEL “CONTACTO CONTROLADO” O FORZADO (2011-PRESENTE)}

En la tercera etapa, las organizaciones indígenas y organizaciones aliadas vienen promoviendo la protección de corredores territoriales o extensiones de bosque conformadas por territorios continuos de pueblos en aislamiento y contacto inicial, mayormente localizados en zonas transfronterizas. Asimismo, el Estado, a través del Viceministerio de Interculturalidad, que es el ente rector de la protección de los pueblos en aislamiento y contacto inicial, ha tenido un papel más activo en el tratamiento de la situación de algunos de ellos. De otro lado, posturas a favor de intensificar el contacto directo con pueblos en aislamiento han sido ampliamente difundidas y llevadas a la práctica.

En efecto, la superposición de una multiplicidad de categorías legales a los territorios de los pueblos en aislamiento y contacto inicial ha provocado

${ }^{4}$ Demanda admitida por la CIDH en el 2007.

${ }^{5}$ Iniciativa que contó con el apoyo financiero de Rainforest Foundation de Noruega.

${ }^{6}$ Organizado por el Alto Comisionado de la ONU para los Derechos Humanos, el Grupo Internacional de Trabajo sobre Asuntos Indígenas, el Viceministerio de Tierras de Bolivia y la Confederación de Pueblos Indígenas de Bolivia 
su fragmentación, imponiendo objetivos y usos que en varios casos implican riesgos para estos pueblos. En respuesta a ello, las organizaciones indígenas, con el respaldo de organizaciones aliadas, nacionales e internacionales, vienen planteando la protección integral de dichos territorios o corredores territoriales. Para ello se busca la articulación, con un enfoque transfronterizo, de los sectores y niveles gubernamentales con los pueblos indígenas y sus organizaciones (Huertas, 2015). Tres corredores han sido fundamentados hasta el momento, dos de ellos en la frontera con Brasil (Yavarí-Tapiche y Pano, Arawak y otros), y uno en la frontera con Ecuador (Napo-Tigre).

De otro lado, no obstante haber sido instalada en el 2009, la Comisión Multisectorial de la Ley 28736, responsable de atender las propuestas de creación de reservas indígenas, empezó a funcionar recién en el 2012, presidida por el Viceministerio de Interculturalidad y teniendo como unidad ejecutora al desaparecido Instituto Nacional de Desarrollo de Pueblos Andinos, Amazónicos y Afroperuano (INDEPA). A partir de entonces, el Estado retomó las gestiones para el establecimiento de las reservas indígenas solicitadas, sin que hasta la fecha ninguna de ellas haya sido creada. En ese contexto, en el 2015, ORAU transfirió al Viceministerio los puestos de control y vigilancia que había construido y mantenido en funcionamiento a lo largo de casi 10 años, buscando proteger las reservas territoriales Mashco Piro, Murunahua e Isconahua.

Desde el 2011, la presencia recurrente de un grupo de mashco piro en aislamiento en un sector de las playas del río Alto Madre de Dios, generó una situación de riesgo debido a su mayor exposición a la interacción física con agentes externos. En julio del 2015, un mes después de la publicación del editorial escrito por Walker y Hill: Protecting isolated tribes para la revista Science, en el que planteaban el "contacto controlado" (2015, p. 1061), la representante del Viceministerio de Interculturalidad anunció públicamente la adopción de esta postura para manejar la situación de los mashco piro. Como consecuencia, generó reacciones de rechazo de parte de organizaciones indígenas, especialistas y la propia Defensoría del Pueblo ${ }^{7}$ ante la falta de fundamentos sólidos que justificaran esa decisión, los riesgos que esta significaba para la vida y salud de la población en aislamiento y la falta de condiciones mínimas en el Estado para afrontar sus consecuencias. Aunque poco después la representante del Viceministerio se retractó ante los medios de comunicación, antropólogos y otras personas vinculadas a esta institución organizaron eventos públicos en los que alentaron impetuosamente el establecimiento de interacciones directas y sostenidas con dicha población ${ }^{8}$. Paralelamente, en el alto Madre de Dios, las

\footnotetext{
${ }^{7}$ Oficio $\mathrm{N}^{\circ}$ 0181-2015-DP/AMASPPI, del 24 de julio de 2015.

${ }^{8}$ Por ejemplo, en el Foro "Avances y Desafíos para la Protección de los Pueblos en Aislamiento" (Lima, 10 de diciembre de 2015), organizado por el Viceministerio de Interculturalidad, y el panel "Indigenous peoples in isolation: terminology, territory and processes of contact" realizado en el XI Congreso de la Society for the Anthropology of Lowland South America (Lima, 20 al 23 de julio de 2017), organizado por antropólogos vinculados al Viceministerio de Interculturalidad.
}

Revista de antropología (marzo 2021) núm. 8: 93 - 104. 
interacciones del Viceministerio de Interculturalidad con la población mashco piro se incrementaron notoriamente, atrayendo a numerosos miembros de este pueblo, agudizando la dependencia de productos introducidos y el riesgo de agresiones (AIDESEP, 2017; CIDH, 2019).

\section{Territorio Y AUTODETERMinaCión}

Como se ha señalado, los principios que orientaron las acciones de las organizaciones indígenas y sus aliados para gestionar la garantía de los derechos de los pueblos en aislamiento desde mediados de los ochenta fueron, de un lado, la seguridad jurídica y la protección territorial y, de otro, el respeto a su forma de vida, conocido también como derecho a la autodeterminación o no contacto forzado. Ambos principios se encuentran estrechamente relacionados, se complementan y se han convertido en una tendencia en el derecho internacional.

La seguridad jurídica de los territorios fue una de las reivindicaciones en torno a las cuales se impulsó el proceso de conformación de organizaciones indígenas amazónicas desde fines de los años sesenta. Su importancia radicaba en la necesidad de asegurar legalmente los territorios indígenas ante el intenso proceso de colonización de la Amazonía promovido como política de Estado, con mayor énfasis desde los años cuarenta. A ello siguió, unas décadas más tarde, la lotización y el otorgamiento de concesiones a favor de empresas para la explotación de los recursos naturales. Estas políticas gubernamentales afectaban a todos los pueblos indígenas, incluyendo a los que se encontraban en aislamiento. A ello se sumaba la rápida expansión de la tala de especies forestales altamente valiosas, como la caoba y el cedro, cuya rentabilidad hacía posible la explotación de estos recursos en lugares de tan difícil acceso como los habitados por pueblos en aislamiento. Cabe recalcar que, como se ha visto líneas arriba, a lo largo de los ańos fueron precisamente madereros, trabajadores de empresas petroleras y misioneros quienes protagonizaron situaciones de contacto forzado con indígenas en aislamiento al ingresar a sus territorios, causándoles graves pérdidas poblacionales y territoriales, así como drásticos cambios en sus patrones de ocupación del espacio y aprovechamiento de los recursos, que deterioraron sus condiciones de vida'.

Por esta razón, desde principios de los años noventa, las organizaciones indígenas contemplaron en sus proyectos el saneamiento físico legal de los territorios de estos pueblos. Ello explica la gestión "temprana", es decir, inaugurando el proceso de defensa y protección de pueblos en aislamiento, dirigida a lograr el establecimiento de reservas territoriales, categoría aplicada en base a lo establecido en la Ley de Comunidades Nativas de 1978. La alta vulnerabilidad de los pueblos en aislamiento a las enfermedades, su rechazo a establecer interacciones sostenidas con la población del entorno y su alta

${ }^{9}$ Como se ha visto, en la Amazonía sur del Perú, este fue el caso de los pueblos nahua, chitonahua y matsigenka. 
dependencia de la flora y fauna de sus territorios, conllevaron a su vez la demanda de prohibir actividades económicas u otras y brindar protección especial a estas áreas.

De otro lado, el respeto al aislamiento como forma de vida, conocido también como el derecho de un pueblo a la autodeterminación o a decidir libre y voluntariamente sobre sus modos de vida, organización, nivel de relación con el entorno, etc., implica que no se debe forzar el contacto o acelerar las interacciones con estos pueblos, dada su decisión de limitarlas, además de sus conocidos efectos sobre su salud y diferentes aspectos de sus vidas. Al respecto, a mediados de los ańos noventa, en el contexto de la defensa de los pueblos en aislamiento del norte de Madre de Dios frente al otorgamiento de una concesión hidrocarburífera en sus territorios, líderes y lideresas de los pueblos harakbut, ese'eja y matsigenka, que solo unas décadas antes habían perdido gran parte de sus poblaciones por la expansión de enfermedades a consecuencia del contacto y la sedentarización forzados, exigieron al gobierno el respeto a la decisión de los pueblos en aislamiento de la región de vivir alejados de la sociedad envolvente y rechazar la injerencia externa en sus territorios. Esta exigencia se ha mantenido a lo largo de los años, convirtiéndose en un principio que ha sido plasmado sobre todo en instrumentos internacionales de defensa de los derechos de los pueblos indígenas.

El derecho a la autodeterminación también implica que si los miembros de estos pueblos optan por incrementar sus interacciones con el entorno, esta opción debería ser respetada, garantizándose su integridad física, sociocultural y territorial, y el espacio y tiempo que la población requiera para desarrollar defensas inmunológicas y mecanismos socioculturales de adaptación a las nuevas y diversas situaciones que el aumento de estas interacciones conllevan. Estamos hablando, en consecuencia, de periodos prolongados de adaptación, lo cual implica una gradualidad en el proceso, quedando claro que este no es unilineal sino que puede revertir, nuevamente, en el aislamiento si la población lo considera mejor, tal como ha ocurrido con segmentos poblacionales mashco piro, mbya guaraní, matsiguenka, etc. Cabe señalar que ninguno de los seis pueblos o colectividades considerados en contacto inicial en el Perú adoptaron esta condición por voluntad o decisión propia; por el contrario, estas atravesaron procesos de contacto forzado por agentes religiosos y económicos, con drásticas pérdidas poblacionales y territoriales así como su desestructuración social y política.

El surgimiento de situaciones de emergencia en salud entre poblaciones en aislamiento también debería ser atendido con los cuidados que amerita y sin la imposición, de parte del personal de salud, de relaciones sostenidas con la población afectada.

La importancia y pertinencia de los principios de respeto al territorio y la forma de vida de los pueblos en aislamiento se ve reflejada en su incorporación 
a normas nacionales e instrumentos internacionales sobre los derechos de los pueblos indígenas. En el ámbito nacional, la Defensoría del Pueblo y el Sector Salud han sido los más claros y contundentes en su adopción. Al respecto, el Informe Defensorial No 101 "Pueblos en Aislamiento Voluntario y Contacto Inicial", aprobado en el año $2005^{10}$, seńala como principales derechos vulnerados de estos pueblos, el derecho a la vida, al territorio y al uso de los recursos, y a la libre determinación. Por su parte, en el 2007, el Centro Nacional de Salud Intercultural del Ministerio de Salud aprobó la norma y guías técnicas de salud para pueblos en aislamiento y contacto inicial. Sus principios rectores son los de: prevención, "Dado que no son posibles intervenciones directas en salud en los pueblos indígenas en aislamiento" (p. 65), y alta vulnerabilidad, ante el alto riesgo de enfermar y morir que representa el contacto (MINSA, 2008, p. 65). Entiéndase el término "enfermar" de acuerdo al significado holístico e integral de salud, el cual comprende las diversas vulnerabilidades (biológicas, sociales, políticas y socioculturales) y no solo la presencia de las enfermedades infecciosas (Neptalí Cueva, comunicación personal, 2019).

De otro lado, la Ley 28736 del sector Cultura (2006) reconoce el derecho de los pueblos en aislamiento al respeto de su decisión en torno a la forma y el proceso de su relación con el resto de la sociedad nacional y con el Estado. También reconoce sus derechos a poseer las tierras que ocupan, restringiendo el ingreso de foráneos a las mismas, el libre acceso a sus tierras y recursos naturales así como el uso extensivo de ellos para sus actividades tradicionales de subsistencia.

En el ámbito internacional, en el 2012 el Alto Comisionado de la ONU para los Derechos de los Pueblos Indígenas publicó las Directrices de Protección de Pueblos en Aislamiento y Contacto Inicial de la Amazonía, el Gran Chaco y la Región Oriental de Paraguay. Este es el principal marco de referencia sobre los estándares internacionales de derechos humanos aplicables a estos pueblos. Los principios y criterios que establece para la protección de su integridad son el respeto y la garantía del derecho a la autodeterminación, entendido como el respeto a su decisión de mantenerse en aislamiento, sin que esto haga presuponer que la situación de estos pueblos no pueda variar; el respeto a sus tierras, territorios y recursos; el respeto a la salud y a la consulta, consentimiento previo libre e informado, este último únicamente en el caso de los pueblos en contacto inicial. De otro lado, plantea la aplicación de protocolos orientados a minimizar las consecuencias de las interacciones directas, si estas se produjeran.

Por su parte, la Declaración Americana sobre los Derechos de los Pueblos Indígenas (2016), señala en su artículo 26 que "los pueblos indígenas en aislamiento voluntario o en contacto inicial tienen derecho a permanecer en dicha condición y de vivir libremente de acuerdo a sus culturas", y que los "Estados adoptarán políticas y medidas adecuadas, con conocimiento y participación de

${ }^{10}$ Aprobado por Resolución Defensorial Nº32-2005-DP. 
los pueblos y las organizaciones indígenas de reconocer, respetar y proteger las tierras, territorios, medio ambiente y culturas de estos pueblos, así como su vida e integridad individual y colectiva”.

\section{Argumentos E Implicancias del ContaCto CONTROLAdo Y DE PROPUESTAS SIMILARES SOBRE LOS PUEBLOS EN AISLAMIENTO Y SUS TERRITORIOS}

Según Walker y Hill (2015), el contacto controlado es la mejor opción para proteger a los pueblos en aislamiento, dadas las dificultades que enfrentan los gobiernos para la protección de los espacios territoriales que estos ocupan. Al respecto, señalan que los gobiernos deberían iniciar "el contacto" con los pueblos en aislamiento, una vez formulado un plan bien organizado, con una estrategia de atención eficaz. Esto significa que la opción de los pueblos en aislamiento de evitar interacciones directas y continuas con la sociedad envolvente y las razones que hayan tenido para ello no se toman en cuenta. Contrariamente, son los funcionarios del gobierno e investigadores vinculados a este los que, bajo una visión etnocéntrica y una práctica colonial, deciden unilateralmente qué se debe hacer con estos pueblos. Se les niega así su derecho a decidir sobre sus propias vidas y su futuro, trastocando severamente todos los aspectos de sus vidas, facilitando la pérdida de sus territorios y abriendo la posibilidad de hacer prevalecer las prioridades gubernamentales sobre ellos y sus territorios.

Los antropólogos mencionados también señalan: "sabemos que pronto, después del contacto pacífico con el mundo de afuera, las poblaciones indígenas sobrevivientes $^{11}$ se restablecen rápidamente del choque poblacional con tasas de crecimiento del $3 \%$ al año. Una vez que el contacto pacífico sostenido ocurre, es más fácil proteger derechos de los nativos que lo que sería para pueblos en aislamiento" (Walker y Hill, 2015, p. 1061). En realidad, los efectos de procesos de contacto forzado o controlado con pueblos indígenas de diferentes países de América del Sur han sido contrarios a lo señalado. En la Amazonía brasileña, durante la segunda mitad del siglo XX, en un contexto de expansión del frente económico y de fomento de la construcción de carreteras a lo largo del territorio nacional, el Estado, a través de la Fundación Nacional del Indio (FUNAI), puso en práctica la política de contacto de los pueblos indígenas como condición para su protección e integración a la comunidad nacional. Los resultados de la política de atracción, contacto y "pacificación" de pueblos indígenas fueron devastadores para estos. Ello derivó en cuestionamientos tanto al interior de la FUNAI como provenientes de organizaciones indígenas, especialistas y otras organizaciones de la sociedad civil. En consecuencia, a fines de los años ochenta se produjo cambios en la política y estrategias de protección de pueblos aislados, adoptándose nuevas directrices. Estos cambios consistieron principalmente en la posición de que ejercer la protección de estos pueblos no significaba necesariamente que

${ }^{11}$ Resaltado por la autora. 
el Estado debía establecer relaciones directas con ellos, sino que a partir de su localización se adoptarían una serie de medidas para salvaguardar sus territorios.

Los pueblos indígenas de la Amazonía peruana han atravesado procesos similares debido a las numerosas muertes que cobraron las enfermedades que se expandieron tras el contacto forzado. Entre los pueblos que afrontaron estas situaciones en la segunda mitad del siglo XX están los harakbut, subgrupos matsiguenka, ese'eja, amahuaca, nahua, etc. Como señala el Ministerio de Salud, la vulnerabilidad de las poblaciones en aislamiento ante el contagio de ciertas enfermedades está ligada en primer lugar al origen exógeno o carácter no endémico de algunos agentes infecciosos. "En el caso de la Amazonía, algunos estudios han contribuido a identificar las enfermedades que posiblemente tengan un origen exógeno. Entre ellas figuran de manera prominente el sarampión, la viruela, influenza A y B, parainfluenza 2 y 3 y el rotavirus" (MINSA, 2003, p. 36). Agrega que diversos autores coinciden en señalar que cualquiera sea la causa de la susceptibilidad ante determinadas enfermedades, poblaciones indígenas que en el pasado han sido vulnerables a enfermedades virales exógenas, requerirían de 3 a 5 generaciones (90 a 150 ańos) para estabilizar su respuesta ante determinado agente infeccioso. Eso explicaría el tremendo potencial mortífero de epidemias reiteradas en tiempos históricos (MINSA, 2003).

Al pretender impulsar un proceso de interacción sostenida para poner a los pueblos en aislamiento bajo observación o "protección" (Walker y Hill, 2015, p. 1061), lo cual implicaría necesariamente su nucleación y sedentarización, la propuesta del contacto controlado impone una forma de vida estrechamente ligada a la alta incidencia de enfermedades ante la concentración de la población en espacios reducidos, favoreciendo el contagio de manera recurrente, de la mano con la disminución de las fuentes de alimento y la contaminación del espacio. En el caso de los pueblos isconahua, chitonahua, mastanahua, nahua y algunos subgrupos matsigenka de la Amazonía peruana, los cuales atravesaron procesos de contacto forzado, su situación de vulnerabilidad inmunológica, sociodemográfica y territorial se expresa de manera pronunciada, colocándolos en condiciones de marcada precariedad (Huertas, 2015; CDC, 2017; AIDESEP, 2017; CIDH, 2019). En todos los casos, su estado de salud es crítico. Las enfermedades infecciosas los afectan de manera recurrente y el sistema de salud no logra atenderlas con eficacia. Como se ha señalado, su alta incidencia está relacionada con los cambios producidos en los patrones de asentamiento y el medio ambiente, específicamente el nucleamiento y la sedentarización, y, con ello, la cercanía de las viviendas, la reducción de las fuentes de agua, la mayor contaminación del espacio vital, el agotamiento de los recursos del bosque para la subsistencia próximos a los poblados, entre otros factores (CDC, 2017).

Este cambio de forma de vida también incide en la prevalencia de anemia y desnutrición, las cuales en gran medida son causadas por la parasitosis favorecida 
por la cercanía de las viviendas, deficientes condiciones de saneamiento (CDC, 2017) y por enfermedades frecuentes en ellos, como la neumonía y la diarrea, que dificultan su adecuada alimentación y recuperación. A las enfermedades infecciosas agudas se suman otras, como la tuberculosis, la hepatitis B e infecciones de transmisión sexual, así como el gran riesgo de infección por VIH y la existencia de altas concentraciones de mercurio en la sangre en la población nahua. Esta realidad demanda una atención efectiva, continua y con pertinencia cultural, que el servicio de salud en el Perú ha demostrado no tener condiciones de brindar (Neptalí Cueva, comunicación personal, 2017). A ello se suma el hecho que, tal como ocurre con los miembros de los pueblos chitonahua y mastanahua, al haber sido desplazados de sus territorios durante el contacto forzado y, por ende, no contar con estos, afronten permanentes dificultades en el abastecimiento de recursos para su subsistencia.

Otro aspecto de la propuesta del contacto controlado es la sobrevaloración de la capacidad del Estado para manejar satisfactoriamente este tipo de situaciones. $\mathrm{Al}$ respecto, la intensificación de las interacciones con los mashco piro en el Alto Madre de Dios entre los ańos 2015 y 2016, donde altos funcionarios y antropólogos del Ministerio de Cultura tuvieron participación, desencadenó situaciones de extremo peligro para ambas partes ante la atracción de numerosos miembros de este pueblo hacia las playas, mayor exposición a agresiones o contactos forzados por otras personas, división y conflictos al interior del grupo por obtener los alimentos, agotamiento y tensión entre los agentes de protección del Ministerio (AIDESEP, 2017; Viceministerio de Interculturalidad, comunicación personal, 2017). En los últimos años, tras las protestas de los representantes de las organizaciones indígenas y otros conocedores, el personal del Estado redujo considerablemente el ritmo de las interacciones con los mashco piro, lo cual, entre otros factores, ha contribuido a disminuir en gran medida el número y frecuencia de su presencia en las playas y, por lo tanto, su exposición a los riesgos del contacto forzado, las enfermedades y las agresiones ${ }^{12}$. Sin embargo, la proximidad al Viceministerio de Interculturalidad de antropólogos interesados en estudiar a los mashco piro, con métodos de investigación directa, podría revertir la situación, con los peligros que ello implica y que ya han sido experimentados por su propio personal.

De otro lado, es necesario reconocer que las deficiencias del Estado en el manejo de situaciones de riesgo por interacciones directas también se extienden al control y protección territorial, tal como afirman Walker y Hill. Los pueblos en aislamiento no solo están amenazados por la presencia de agentes económicos dedicados a actividades ilícitas al interior de reservas indígenas/territoriales creadas o en trámite, sino que, además, tal como ocurre en la Reserva Kugapakori Nahua Nanti y otros, por la superposición de concesiones hidrocarburíferas, lo

${ }^{12}$ Esto no significa que la situación no pueda cambiar, tomando en cuenta la persistencia de ciertos grupos en establecer un contacto sostenido con los mashco piro, principalmente con fines religiosos y de investigación antropológica. 
cual es permitido por la propia legislación nacional, mostrando así el peso que tiene esta actividad con relación a los derechos fundamentales de los pueblos en aislamiento. Sin embargo, estas limitaciones en el control territorial de ninguna manera justifican la ejecución del contacto controlado, planteado por dichos antropólogos, sino que debieran conllevar el fortalecimiento de los mecanismos de protección del territorio.

\section{Algunas PaUtas PARA ENFRENTAR SituaCiONES DE RIESGO POR INCREMENTO DE INTERACCIONES}

Es necesario que el Estado y la sociedad civil, en particular el sector académico, adopten políticas y prácticas respetuosas de la forma de vida y los derechos fundamentales de los pueblos indígenas en aislamiento. Ello implica garantizar el ejercicio de su derecho a la autodeterminación, brindar la seguridad jurídica y protección eficiente a sus territorios, prohibiendo el otorgamiento de derechos en favor de terceros en su interior. Paralelamente, es necesario implementar estrategias de prevención epidemiológica $y$, en aquellos casos donde existen tensiones entre estos pueblos y las poblaciones vecinas, estrategias para evitar conflictos. Estas son condiciones básicas para garantizar su sobrevivencia física y sociocultural.

En términos más concretos, se requiere personal especializado, logística y presupuesto para reforzar la protección de los territorios de estos pueblos, implementando sistemas de vigilancia, identificando y erradicando oportunamente las presiones y las amenazas. Se necesita escuchar más a la población que comparte territorios con pueblos en aislamiento, la cual es mayoritariamente indígena, a fin de identificar conflictos potenciales y adoptar medidas idóneas para evitar que estos se produzcan. Las tensiones se pueden dar por presiones externas, interacciones hostiles con dichos pueblos, escisiones o conflictos intraétnicos, entre otros. La coordinación intersectorial y con las poblaciones locales con fines preventivos y de atención de emergencias por exposición y riesgo de expansión de enfermedades parece ser uno de los mayores desafíos para el Estado.

Ante situaciones de riesgo de interacciones directas que involucren a poblaciones en aislamiento, es necesario descartar la posibilidad de que estas sean manipuladas o interpretadas en favor de intereses académicos, religiosos, económicos o gubernamentales, y anteponer la garantía de la integridad y los derechos fundamentales de la población mencionada. Las instituciones involucradas, entre ellas las académicas, deberían establecer códigos de ética y mecanismos para asegurar que sus prácticas o acciones no atenten contra los principios establecidos en las normas nacionales e internacionales referidas a estos pueblos. 
De presentarse el caso de alguna población que realmente busque ir incrementando la frecuencia de sus interacciones con el entorno, el Estado debería estar preparado para garantizar mínimamente las condiciones sanitarias, la integridad personal y grupal, sus territorios y fuentes de alimento, y el tiempo que sea necesario para que desarrollen los mecanismos inmunológicos, psicológicos y socioculturales que requieran para adaptarse a los cambios y a las nuevas situaciones que enfrentarán. En suma, se trata de generar las condiciones para que, si se diera el caso, el incremento de las interacciones con la población del entorno no desencadene los impactos negativos que este ha tenido históricamente y que han llevado a varios pueblos indígenas al riesgo de extinción.

\section{CONSIDERACIONES FINALES}

El contacto controlado o forzado, o la postura en favor de que funcionarios, antropólogos u otras personas tomen la iniciativa de establecer relaciones directas y sostenidas con poblaciones que las rechazan, como las que se encuentran en aislamiento, encierra actitudes, políticas y prácticas históricamente cuestionadas y en algunos países prohibidas debido a la imposición de formas de vida y con ello la falta de respeto a las decisiones y diferencias culturales, además de los graves efectos que han tenido sobre estos pueblos.

Como se ha visto, contrariamente a lo que han señalado los impulsores del contacto controlado y de planteamientos similares, el aislamiento no se fomenta, sino que es una realidad producto de la decisión de pueblos indígenas o segmentos de estos. Lo que se promueve es el respeto hacia esta decisión y, con ello, al derecho de estos pueblos a decidir sobre su forma de vida, organización y nivel de relacionamiento con el entorno. De la misma manera, las gestiones para el reconocimiento y la protección territorial no tienen el objetivo de encerrar a estos pueblos para mantenerlos al margen de la sociedad envolvente, algo que, por cierto, además de representar una imposición, sería inviable, sino garantizar sus territorios como base material e inmaterial de su existencia. A la vez, con la demanda al Estado de que implemente mecanismos de protección y prohíba la ejecución de actividades económicas o de cualquier otra índole dentro de sus territorios, se busca evitar que estos pueblos sigan estando expuestos a procesos de desaparición física y cultural, los cuales históricamente han estado vinculados a la ampliación de la frontera económica.

Los principios de respeto a la autodeterminación y al territorio, adoptados en base a evidencia empírica que ha demostrado dramáticamente los efectos del contacto forzado sobre los pueblos indígenas, han sido acogidos en instrumentos internacionales de defensa y protección de los derechos de los pueblos indígenas.

La posición en favor del "contacto controlado" también sobrevalora la capacidad y condiciones del Estado de manejar satisfactoriamente los efectos 
de los contactos forzados. La grave situación de todos los pueblos indígenas en contacto inicial, en términos de salud, territorio y subsistencia, muestra las notorias carencias del Estado en este sentido. De otro lado, es innegable que el Estado también presenta serias limitaciones para consolidar la protección de las reservas indígenas y otras áreas habitadas por pueblos en aislamiento. Sin embargo, contrariamente al abandono de estas acciones, lo cual se desprende de la propuesta de Walker y Hill para proceder a organizar el contacto controlado, el Estado tiene la responsabilidad de reforzar la protección. Ello incluye el incremento del presupuesto institucional, una mayor articulación con los sectores y niveles gubernamentales que comparten responsabilidades de protección, así como una mayor coordinación con las comunidades y organizaciones indígenas que vienen ejerciendo esta protección desde hace décadas.

El anuncio de la adopción del "contacto controlado" por el Estado peruano, de manera casi inmediata a la difusión de este planteamiento a mediados del 2015 en una revista de investigación científica, tornó esta propuesta en aún más peligrosa al ser asumida como una política y una práctica estatal, que por sus consecuencias sobre los pueblos en aislamiento había sido descartada previamente en otros países. El rechazo de la sociedad civil a dicho anuncio tuvo un efecto positivo al lograr una rectificación verbal de parte del Estado; sin embargo, la intensificación de las interacciones directas y sus consecuencias en la práctica, el afán de sus funcionarios y asesores de justificar la decisión inicial en eventos públicos posteriores y, en términos generales, lo propicia que es esta postura para la liberación de los territorios de los pueblos en aislamiento en favor de la ampliación de la frontera económica, demuestran que el contacto controlado o forzado sigue siendo una amenaza potencial para estos pueblos.

Contrariamente a lo sugerido por los impulsores del "contacto controlado" o forzado respecto a una supuesta falta de idoneidad en la postura en favor de no forzar interacciones con pueblos en aislamiento, de no haberse producido avances en el reconocimiento de ese principio, que se traduce como respeto a la autodeterminación y el territorio de los pueblos en aislamiento, muy probablemente varios de ellos habrían sido diezmados o se habrían extinguido por el efecto de las epidemias y los conflictos, o se habrían desplazado y la mayor parte de sus territorios estaría ocupada por concesiones madereras, más áreas de explotación petrolera, cocaleros y narcotraficantes, atravesados por carreteras y caminos forestales.

\section{REFERENCIAS}

Álvarez Lobo, Ricardo (1998). Sepahua V - Campo de misión, tomo 7. Lima: Colección Antisuyo. 
Asociación Interétnica de Desarrollo de la Selva Peruana [AIDESEP] (2017). Situación de los pueblos en aislamiento y contacto inicial de la Amazonía peruana (informe sin numeración). Lima.

Centro Nacional de Epidemiología, Prevención y Control de Enfermedades [CDC] (2017). Análisis de la situación de salud del pueblo nahua de Santa Rosa de Serjali en la Reserva Territorial Kugapakori Nahua Nanti y otros (informe ministerial sin numeración). Lima.

Comisión Interamericana de Derechos Humanos [CIDH] (2019). Situación de los derechos humanos de los pueblos indígenas y tribales de la Panamazonía. Aprobado por la Comisión Interamericana de Derechos Humanos el 29 de setiembre (documento 176). Recuperado de http://www.oas.org/es/cidh/ informes/pdfs/Panamazonia2019.pdf

Chirif, Alberto y García, Pedro (2007). Marcando territorio: Progresos y limitaciones de la titulación de territorios indígenas en la Amazonía. Lima: Grupo Internacional de Trabajo sobre Asuntos Indígenas [IWGIA].

Helberg, Heinrich y Reynoso, Patricia (1986). Primer estudio etnográfico del grupo étnico yura o nahua (documento de trabajo). Lima.

Huertas, Beatriz (2002). Los pueblos indigenas en aislamiento: Su lucha por la sobrevivencia y la libertad. Lima: Grupo Internacional de Trabajo sobre Asuntos Indígenas [IWGIA].

Huertas, Beatriz (2012). Despojo territorial, conflicto social y exterminio. Pueblos indigenas en aislamiento, contacto esporádico y contacto inicial de la Amazonía peruana. Lima: Grupo Internacional de Trabajo sobre Asuntos Indígenas [IWGIA], Instituto de Promoción de la Economía Social [IPES].

Huertas, Beatriz (2015). Corredor territorial de pueblos indigenas en aislamiento y contacto inicial pano, arawak y otros. Diagnóstico y fundamentos antropológicos. Lima: Asociación Interétnica de Desarrollo de la Selva Peruana [AIDESEP].

Ministerio de Salud [MINSA] (2003). Pueblos en situación de extrema vulnerabilidad: El caso de los nanti de la reserva territorio Kugapakori Nahua, río Camisea, Cusco. Lima: Ministerio de Salud, Oficina General de Epidemiología.

Ministerio de Salud [MINSA] (2008). Norma y guías técnicas en salud. Indígenas en aislamiento y contacto inicial. Lima: Ministerio de Salud, Instituto Nacional de Salud.

Shepard, Glenn H. Jr. (2016). Ceci n'est pas un contacte: The fetishization of isolated indigenous people along the Peru-Brazil border. Tipiti: Journal of the Society for the Antrhopology of Lowland South America, 14(1), 135-137.

Shinai Serjali (2004). Aqui vivimos bien. Kamyeti notimaigzi aka: Territorio y uso de recursos de los pueblos indigenas de la Reserva Kugapakori Nahua. Lima: Shinai Serjali. 
La importancia de la ética, el respeto a la autodeterminación y los derechos fundamentales de los pueblos indígenas en aislamiento en la antropología

Walker, Robert y Hill, Kim (2015) Protecting isolated tribes. Science, 348(6239), 1061.

Zarzar, Alonso (1983). Las relaciones tribales en el bajo Urubamba y alto Ucayali. Lima: Centro de Investigación en Promoción Amazónica [CIPA]. 